\title{
Perfil de la Lluvia Ácida en la Ciudad de Quito (Ecuador) Durante los Meses de Diciembre-2008 y Enero-2009
}

\section{RONNY FLORES $1 *$, PABLO BONILLA 2}

' Laboratorio de Química Ambiental, Facultad de Ciencias Químicas, Universidad Central del Ecuador, Quito

2 Laboratorio de Química Analítica, Facultad de Ciencias Químicas, Universidad Central del Ecuador, Quito

*Correspondencia: ronnyflores@correo.unam.mx

\section{Resumen}

El presente trabajo de investigación analizó la composición de la lluvia en la ciudad de Quito, capital del Ecuador, durante los meses de diciembre de 2008 y enero de 2009. Se distribuyeron 17 sitios de muestreo a lo largo de la ciudad. Se midió en el agua de lluvia el pH, conductividad, calcio, nitratos y sulfatos. Se encontró que aproximadamente el 50 \% de superficie de la ciudad está afectada por lluvia ácida, es decir, con lluvia con un $\mathrm{pH}$ menor a 5,6. Lo que indica que la atmósfera de Quito está contaminada de óxidos de azufre y nitrógeno, provenientes de las plantas termoeléctricas y del tráfico vehicular, que por oxidación se han convertido en ácido sulfúrico y nítrico, respectivamente, que han acidificado el agua de lluvia. La lluvia ácida es periudicial para los ecosistemas acuáticos y terrestres.

Palabras claves.- Lluvia ácida, contaminación, ciudad de Quito, pH, atmosfera.

\section{Abstract}

The present work analyzed Quito city rain's composition during December 2008 and January 2009 months. Seventeen sampling places were distributed along in the city. In rainwater were analyzed: $\mathrm{pH}$, conductivity, calcium, nitrates and sulfates. Approximately $50 \%$ of surface in the city is affected by acid rain (rain with minor $\mathrm{pH}$ of 5,6$)$. What indicates that the atmosphere of Quito is contaminated with sulfur and nitrogen oxides, coming from thermoelectric plants and traffic. They become sulfuric and nitric acids, respectively, and they acidified rainwater. The acid rain is harmful for aquatic and terrestrial ecosystems.

Key words: Acid rain, contamination, Quito city, pH, atmosphere.

\section{Introducción}

Conocer la calidad del aire de la ciudad es importante para garantizar el bienestar de los ciudadanos y para que las autoridades tomen decisiones para mejorarlo. La lluvia es un mecanismo natural de limpieza de la atmosfera que remueve gases atmosféricos, aerosoles, partículas y todos aquellos compuestos que han sido introducidos a la atmósfera por diferentes fuentes de emisión naturales (erupciones volcánicas, incendios forestales) o antropogénicas (plantas de energía, industria y transporte). Por lo tanto, la lluvia es un indicador útil de los niveles de contaminación ambiental, que aporta datos valiosos acerca de entradas de compuestos tóxicos a los sistemas terrestres y acuáticos [1]. La precipitación en el Distrito Metropolitano de Quito (DMQ) observa un régimen bimodal, presentando el primer periodo lluvioso entre febrero y mayo y el otro durante octubre y diciembre. Precipitaciones mínimas se observan principalmente entre junio a agosto. En la ciudad de Quito, capital del Ecuador, el aire puede estar contaminado por las emisiones de los vehículos livianos y pesados, seguidas por las fuentes fijas, principalmente las centrales termoeléctricas, y por las fuentes aéreas, como las canteras y la vegetación [2]. Una de las consecuencias de la contaminación atmosférica es la formación de la lluvia ácida. El agua de lluvia en ausencia de contaminantes 
atmosféricos es ligeramente ácida, tiene un $\mathrm{pH}$ de 5,6, debido a la disolución del $\mathrm{CO}_{2}$ atmosférico de acuerdo a las siguientes reacciones [3]:

$$
\begin{array}{ll}
\mathrm{CO}_{2}+\mathrm{H}_{2} \mathrm{O}=\mathrm{HCO}_{3}^{-}+\mathrm{H}^{+} & \mathrm{K}_{\mathrm{al}}=4,45 \times 10^{-7} \\
\mathrm{HCO}_{3}{ }^{-}=\mathrm{CO}_{3}{ }^{2-}+\mathrm{H}^{+} & \mathrm{K}_{\mathrm{a} 2}=4,69 \times 10^{-11}
\end{array}
$$

Si además en el aire están presentes gases contaminantes como $\mathrm{SO}_{2}$ y $\mathrm{NO}$ estos se disuelven en el agua y bajan aún más el pH del agua lluvia por la formación de ácido sulfúrico y nítrico, por oxidación de los óxidos respectivos. Se conoce, entonces, como lluvia ácida a la lluvia que tiene un pH inferior a 5,6. La medición del $\mathrm{pH}$ de las precipitaciones ayuda a determinar el efecto de la lluvia sobre el suelo, la vegetación y los lagos [4]. La especie principal que inicia la secuencia de reacciones es el radical hidroxilo [5], formado por reacciones fotoquímicas en la atmósfera:

$$
\begin{aligned}
& \mathrm{SO}_{2}+\mathrm{HO} \bullet=\mathrm{HSO}_{3} \bullet \\
& \mathrm{HSO}_{3} \bullet+\mathrm{O}_{2}=\mathrm{SO}_{3}+\mathrm{HO}_{2} \bullet \\
& \mathrm{SO}_{3}+\mathrm{H}_{2} \mathrm{O}=\mathrm{H}_{2} \mathrm{SO}_{4} \\
& 2 \mathrm{NO}+\mathrm{O}_{2}=2 \mathrm{NO}_{2} \\
& \mathrm{NO}_{2}+\mathrm{HO} \bullet=\mathrm{HNO}_{3}
\end{aligned}
$$

Los contaminantes primarios de la atmósfera son los óxidos de azufre y nitrógeno formados directamente de las fuentes de combustión y estos contaminantes al trasformase en la atmósfera a los ácidos respectivos se convierten en contaminantes secundarios. El petróleo contienen pequeños porcentajes de compuestos de azufre, en consecuencia, los óxidos de azufre aparecen como subproductos indeseables de la combustión de combustibles fósiles. Los óxidos de azufre se eliminan del aire mediante conversión en ácido sulfúrico y sulfatos. Y terminan depositándose en la tierra [6]. La mayor fuente de óxidos de azufre la constituyen las fuentes fijas (rellenos sanitarios, termoeléctricas e industrias) con $85,5 \%$ y en menor porcentaje las fuentes móviles 14,5 $\%$. En las fuentes fijas el $42,8 \%$ corresponde a las centrales termoeléctricas y el $27,3 \%$ a hornos y calderos industriales. Las fuentes móviles (buses y vehículos pesados a diesel y vehículos livianos a gasolina aportan el 10,5\% [7]. La quema de combustibles fósiles se realiza en presencia de aire que está formado aproximadamente de un $78 \%$ de nitrógeno y un $21 \%$ de oxígeno
[3]. Durante la combustión el nitrógeno del aire se oxida y forma óxidos debido a la alta temperatura de la combustión. En la atmósfera, los óxidos se convierten en ácido nítrico y nitratos que se depositan en el suelo [6]. La mayor parte de los óxidos de nitrógeno artificiales $\mathrm{NO}$ ( $\mathrm{NO}+\mathrm{NO}_{2}$ ) se derivan de las fuentes móviles (tráfico vehicular y aéreo) con $53,8 \%$, de estos los buses y vehículos pesados a diesel aportan el $28,6 \%$, particulares, taxis y camionetas a gasolina el $21,6 \%$. Las fuentes fijas generan el $44,5 \%$ siendo las centrales termoeléctricas las principales con el 37,8\% [7]. Por lo tanto, la lluvia ácida tiene su origen en centros urbanos e industriales, que son las principales áreas de emisión de sus precursores: óxidos de azufre y nitrógeno. Los cuales son trasportados a cientos o miles de kilómetros de sus fuentes [1].

La lluvia ácida tiene efectos negativos sobre los ecosistemas terrestres y acuáticos. Para la salud, los riesgos potenciales se relacionan con la exposición continua de los óxidos de azufre y nitrógeno como efectos respiratorios en animales y humanos. La lluvia ácida puede producir efectos indirectos, ya que las aguas acidificadas pueden disolver metales y sustancias tóxicas del suelo, rocas, conductos y tuberías. La lluvia ácida acelera el deterioro de los materiales de construcción y pinturas incluyendo construcciones históricas, estatuas y esculturas. Los efectos en el suelo por la lluvia ácida depende de la cantidad total de lluvia en el área y de sus características. Algunos suelos contienen compuestos neutralizantes de ácidos como carbonatos de calcio. La Iluvia ácida puede provocar la pérdida de los compuestos alcalinos calcio y magnesio, que son nutrientes importantes para la biota, lo cual origina empobrecimiento por la disminución de la fertilidad y la productividad. La lluvia ácida puede liberar el aluminio del suelo que es tóxico para las plantas y daña las raíces de los árboles. En la vegetación sensible es frecuente el daño en el follaje, con la caída prematura de las hojas, aumentando la susceptibilidad a plagas y enfermedades por excesiva concentración de ácido o de los gases $\mathrm{SO}_{2}$ y $\mathrm{NO}_{x}$. Las plantas se debilitan haciéndose más susceptibles al frío y la sequía. La lluvia ácida, también, causa la acidificación de lagos y corrientes que genera serios efectos en la constitución química del agua, provocando un aumento en la concentración de elementos tóxicos como los iones aluminio que pueden afectar a muchos tipos de peces; además, de interrumpir ciclos reproductivos de plantas acuáticas y animales [1], [3] y [4]. 
No existe un estudio formal en la ciudad de Quito sobre la lluvia ácida. Desde el año 2004 la Corporación Municipal para el Mejoramiento del Aire de Quito (CORPAIRE: www.corpaire.org) se encarga del monitoreo de los contaminantes primarios como los óxidos de azufre y nitrógeno [7]; pero, no de los contaminantes secundarios como los componentes de la lluvia ácida; tampoco, la normativa ecuatoriana para la calidad del aire ambiente incluye dentro de sus parámetros de control a la lluvia ácida [8]. El presente estudio investiga si la ciudad de Quito tiene problemas de lluvia ácida. Además, busca involucrar a la ciudadanía en cuestiones ambientales con la participación activa de estudiantes.
Este estudio servirá de base para un proyecto mayor que involucrará a una población más amplia y durante un periodo mucho más largo.

\section{Materiales y Métodos}

\subsection{Toma de muestras}

La recolección de las muestras de agua lluvia la realizaron 17 alumnos de la Facultad de Ciencias Químicas de la Universidad Central del Ecuador, distribuidos en diferentes sectores de la ciudad de Quito, de acuerdo con las parroquias en donde viven (ver Figura 1).

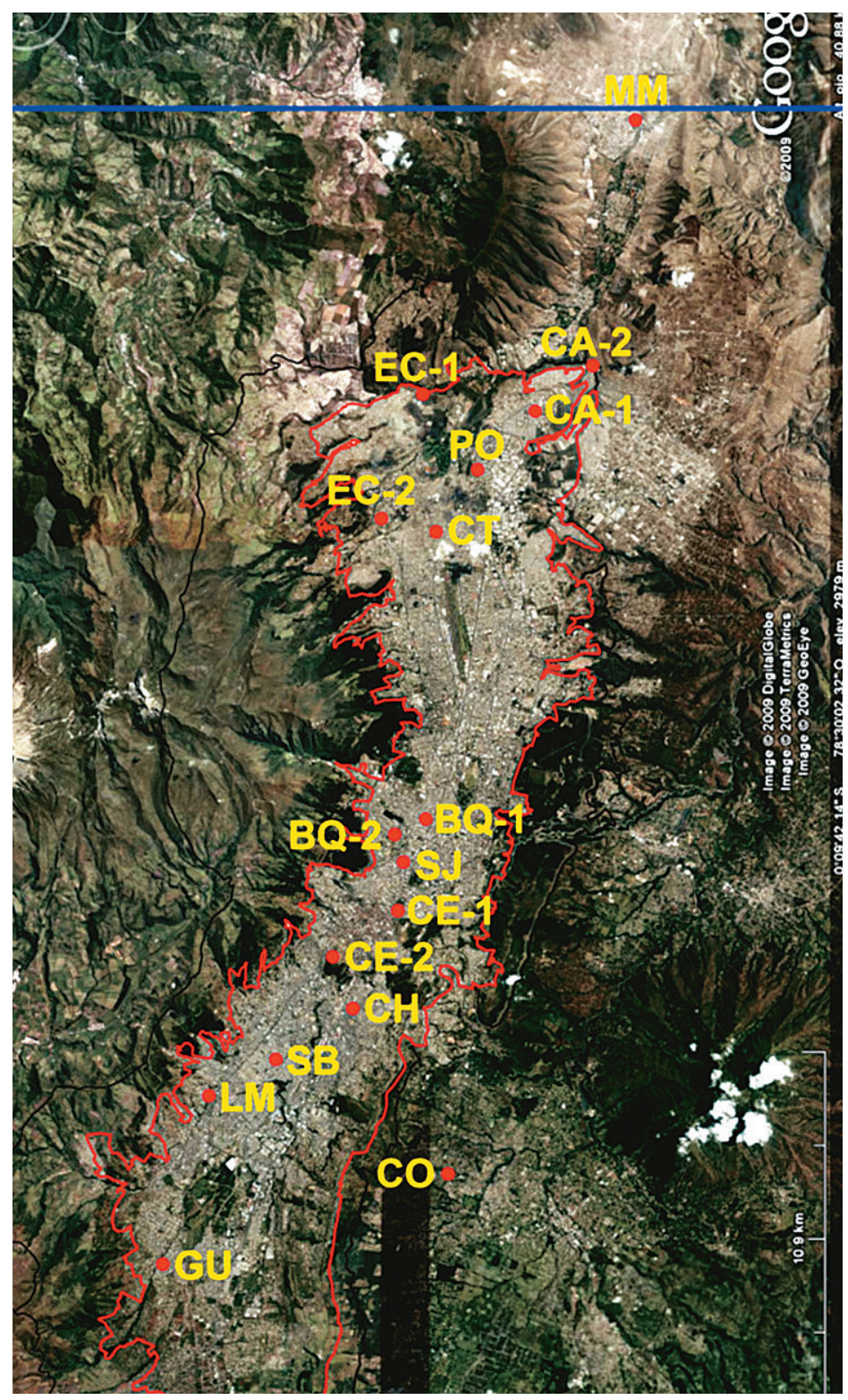

CLAVE:

MM: MITAD DEL MUNDO
CA: CARCELÉN
EC: EL CONDADO
PO: PONCEANO
CT: COTOCOLLAO
BQ: BELISARIO QUEVEDO
SJ: SAN JUAN
CE: CENTRO HISTÓRICO
CH: CHIMBACALLE
SB: SAN BARTOLO
LM: LA MENA
CO: CONOCOTO
GU: GUAMANÍ

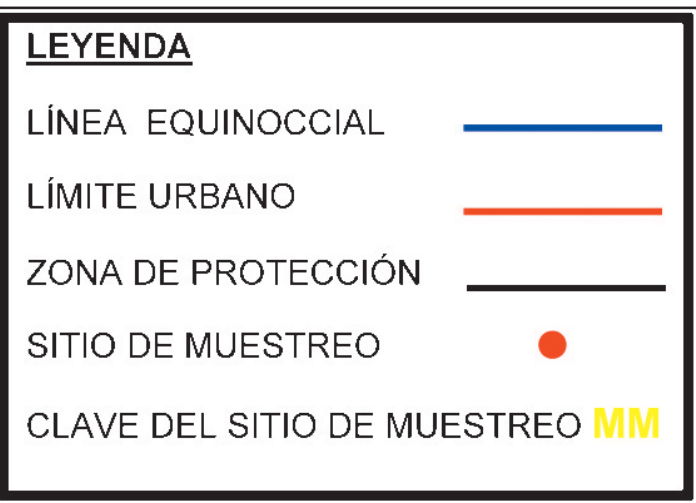

Figura 1. Foto satelital de la ciudad de Quito con los 17 sitios de muestreo. (Foto modificada del Google Earth) 
El perfil de la lluvia puede variar significativamente de un lugar a otro en distancias menores a $10 \mathrm{~km}$; una lluvia puede ser grande y cubrir toda una región mientras que otras son pequeñas de sólo $10 \mathrm{~km}$ o menos; entonces, para obtener una visión real hay que tomar mediciones en diferentes lugares, ya que el $\mathrm{pH}$ puede variar de un sitio a otro o puede cambiar durante el curso de una tormenta [4]. Con la ayuda del programa informático Google Earth 5 (http://earth.google.es) se determinaron las coordenadas de los sitios de muestreo.

A cada voluntario se le proveyó de cinco frascos de vidrio color ámbar de $100 \mathrm{~mL}$ de capacidad, un embudo de plástico grande y una probeta de plástico de 100 $\mathrm{mL}$. Y, tiras de papel indicador de $\mathrm{pH}$ que cambian de color marca Precision pH 4070 Test Strip con un rango de $\mathrm{pH}$ de 4,0 a 7,0 con intervalo de 0,4 unidades de $\mathrm{pH}$. Para la toma de muestras se utilizó el protocolo del Programa Global Learning and Observations to Benefit the Environment [4]. Se les instruyó para que colocaran la probeta con el embudo en un lugar abierto y despejado alejado por lo menos tres metros de cualquier construcción y a $50 \mathrm{~cm}$ del suelo. Luego de finalizada la lluvia debían medir el volumen recolectado y el pH de la muestra. Las muestras se guardaban en la refrigeradora, dentro del frasco ámbar etiquetado, hasta ser enviadas al Laboratorio de Química Ambiental de la Facultad de Ciencias Químicas para el resto de análisis. Las muestras de agua lluvia se recolectaron durante los meses de diciembre de 2008 y enero de 2009. La superficie de la ciudad de Quito analizada comprende aproximadamente un rectángulo de 33,85 por 13,69 $\mathrm{km}$ de lado, es decir, un área de 463,4 km².

\subsection{Análisis de muestras}

Durante el tiempo que duró el análisis, las muestras estuvieron almacenas a $4{ }^{\circ} \mathrm{C}$ de temperatura. La conductividad se midió con un equipo SevenEasy Conductivity de Mettler Toledo, Los sulfatos se determinaron por el método turbidimétrico $4500-\mathrm{SO}_{4}{ }^{2-} \mathrm{E}$ [9] en un equipo espectrofotométrico Genesys 10UV de Thermo Electron Corporation $y$, los nitratos por el método de reducción de la hidracina $4500-\mathrm{NO}_{3}-\mathrm{H}$, [9] en un equipo espectrofotométrico Genesys 10UV de Thermo Electron Corporation. Todos los reactivos utilizados fueron grado PA. Calcio, magnesio y hierro se determinaron por espectroscopia de absorción atómica en un equipo Perkin Elmer modelo AAnalyst 200.
Para los gráficos de contorno se utilizó el programa Surfer versión 8,01 Surface Mapping System de Golden software. Los límites de la ciudad de Quito y sus parroquias se obtuvieron de la base de datos del Sistema de Información Geográfico SavGIS versión 9 (http://www.savgis.org/es) desarrollado por el Institut de Recherche pour le développement (IRD) utilizado por la Dirección Metropolitana de Planificación Territorial de Quito. Y, la fotografía satelital de la ciudad de Quito con los lugares de muestreo se realizó con el programa Google Earth 5.

\section{Resultados y Discusión}

Las muestras de lluvia se recolectaron a lo largo de la ciudad de Quito desde Guamaní al sur hasta la Mitad del Mundo al norte. Fueron 17 sitios de muestreo lo que permitió obtener una visión amplia de la composición de la lluvia en Quito. Los datos recolectados durante un mes fueron promediados y el valor promedio junto con la longitud y latitud del sitio de muestreo correspondiente se ingresó en el programa Surfer para construir los gráficos de contorno o isolíneas. Para el análisis espacial se utilizó el modelo de interpolación kriging [10]. Los resultados para los diferentes analitos se muestran en las figuras 2, 3, 4, 5 y 6 . La interpretación de los gráficos obtenidos se dificulta toda vez que los contaminantes pueden viajar muchos kilómetros del lugar de la emisión acarreados por el viento; sin embargo, sí se puede obtener información útil para determinar la magnitud de la contaminación en la ciudad de Quito. Se consideran desfavorables para la dispersión atmosférica de los contaminantes en Quito, a la topografía que puede favorecer el atrapamiento de contaminantes en rincones, al régimen de los vientos que poco contribuye a la circulación del aire sobre todo en el Centro Histórico, a la insolación que propicia la formación de contaminación fotoquímica y a las inversiones térmicas que contribuyen a bloquear el aire a nivel del suelo. En cuanto a los vientos, en Quito, se ha establecido que las direcciones dominantes y velocidades promedio son muy variables según las estaciones y el espacio. De manera general, no hay vientos fuertes. Cuando soplan hacia el norte, en el sentido del valle, permiten ocasionalmente una buena dispersión de los contaminantes por un efecto de barrido. Sin embargo, las condiciones atmosféricas más comunes provocan el encuentro de masas de aire provenientes del sur con otras provenientes del norte a nivel del Panecillo, produciéndose remo- 
linos, fricciones de masas de aire y finalmente la permanencia de los contaminantes en esa zona del Centro Histórico [11].

En general, la conductividad, nitratos y sulfatos mostraron valores más altos durante el mes de diciembre en comparación con el mes de enero. Esto podría ser debido al fenómeno de la inversión térmica que se produce a una altura de aproximadamente $500 \mathrm{~m}$ por encima del suelo. Esta situación es visible principalmente en el centro y en la parte noroccidental de la ciudad y se revela más frecuentemente en la madrugada hasta las 10 ol 1 a.m. Las condiciones atmosféricas estables que caracterizan a estos períodos impiden una buena dispersión de los contaminantes y favorece el estancamiento temporal de los contaminantes en el aire, principalmente en los meses de verano (julio, agosto y septiembre) y en el Veranillo del Niño en noviembre y diciembre [7], lo que podría explicar porque la contaminación fue mayor en diciembre que en enero.

El pH es considerado como el principal indicador de la lluvia ácida, depende de la cantidad de lluvia y la presencia de polvo atmosférico. Los datos fueron obtenidos in situ en los meses de diciembre de 2008 y enero de 2009 (ver Figura 2) allí se aprecia que aproximadamente el $50 \%$ de la superficie en estudio tiene un $\mathrm{pH}$ menor a 5,6; es decir, hay presencia de lluvia ácida con un mínimo de 4,6 unidades en la región oriental de la ciudad. El pH es más básico al occidente de Quito en comparación con el oriente que se podría explicar porque la vegetación de la ladera del Pichincha al occidente ayuda a neutralizar los contaminantes [11] haciendo la lluvia más básica.

La conductividad del agua lluvia está directamente relacionada con la presencia de iones disueltos en la misma. En la Figura 3 se aprecia que los valores más altos están hacia el norte de la ciudad que se explicaría por la presencia de canteras en la zona norte de Quito que generan material particulado, además, de la erosión del suelo y la poca vegetación que generan polvo que contribuye a aumentar la conductividad. También influye la escasez de lluvias del lugar, pues se conoce que en la ciudad de Quito hay una disminución de la precipitación de sur a norte y de las pendiente más elevadas del Pichincha hacia las más bajas [11].
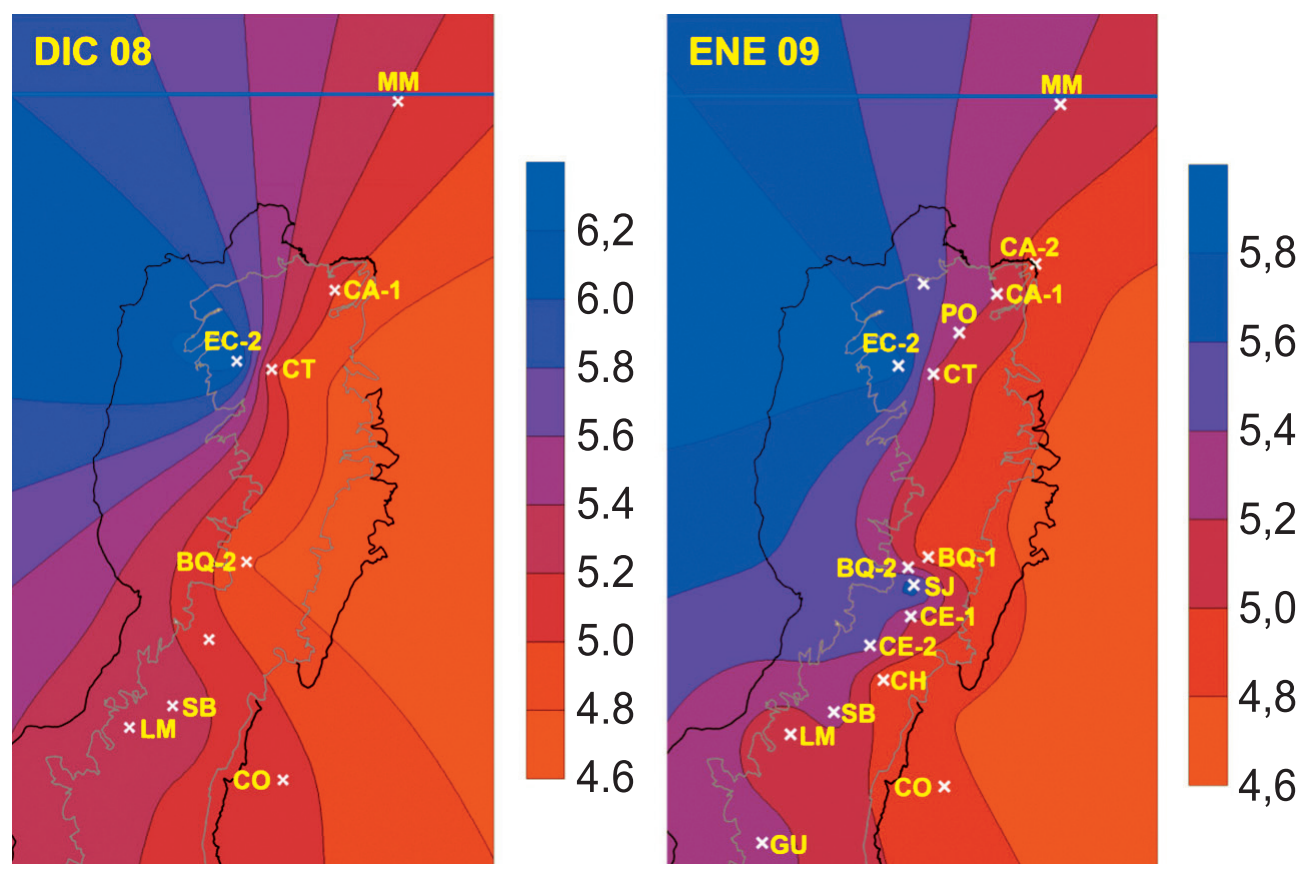

Figura 2. Gráfico de contorno para el pH del agua de lluvia en la ciudad de Quito durante los meses de diciembre de 2008 y enero de 2009. Datos promedio de cada mes. 

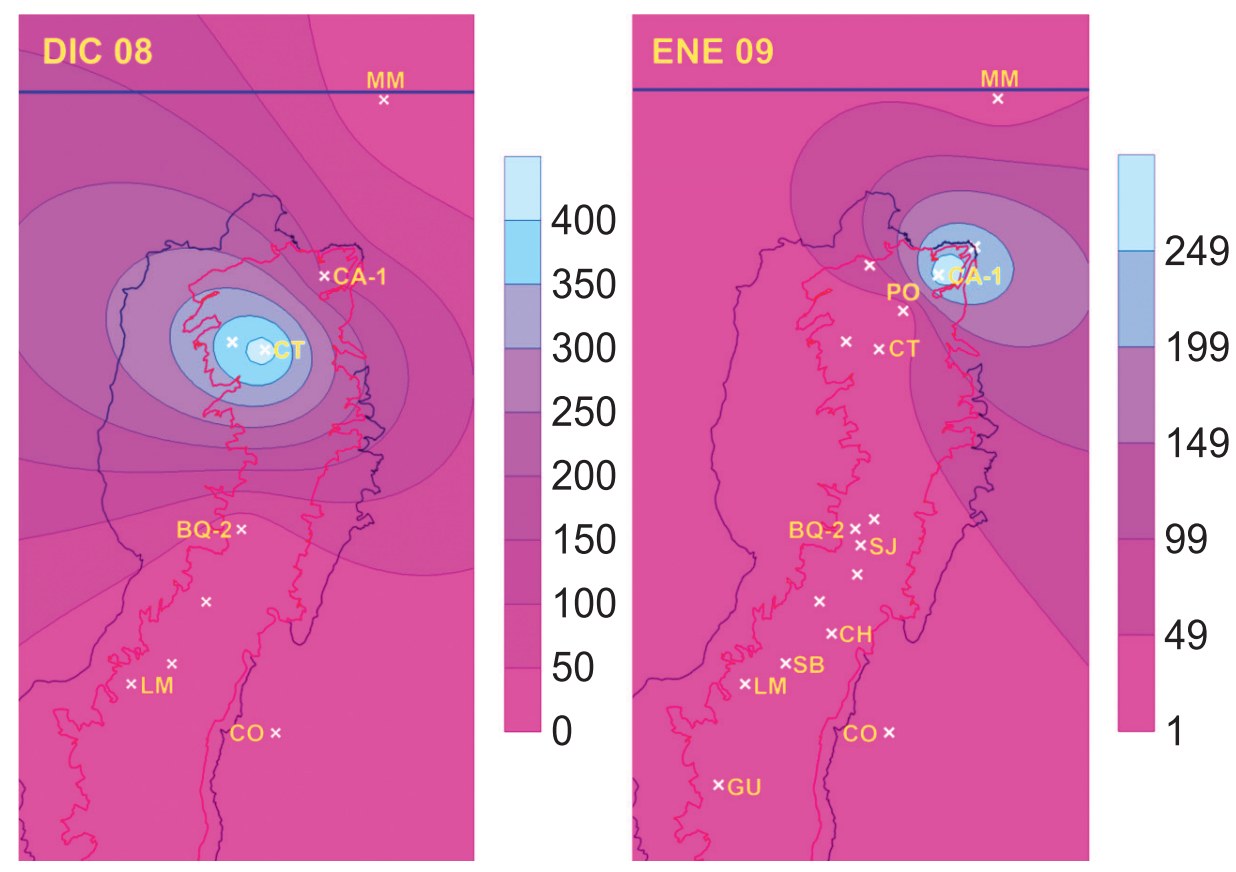

Figura 3. Gráfico de contorno de la conductividad, en uS/cm, del agua de lluvia en la ciudad de Quito durante los meses de diciembre de 2008 y enero de 2009. Datos promedio mensuales.

El calcio es el principal elemento neutralizador de los compuestos ácidos de la lluvia y tiene su origen en las rocas que son utilizadas para la construcción [1]. El calcio está directamente relacionado con la presencia de partículas aéreotransportadas provenientes de los suelos erosionados, áreas sin cubierta vegetal, calles sin pavimentar y canteras por lo que, al igual que la conductividad, presenta los valor más altos en la zona norte de la ciudad Quito (ver Figura 4).
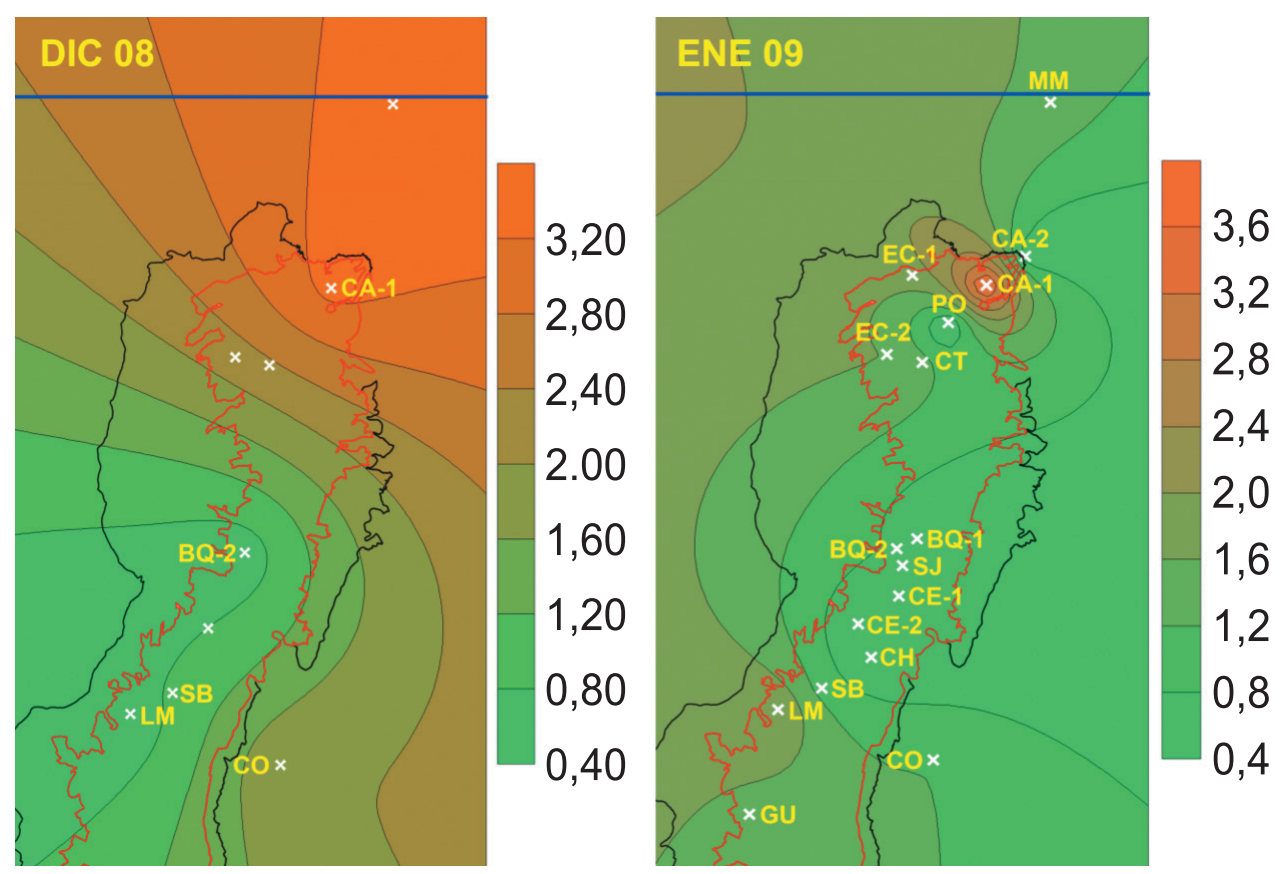

Figura 4. Gráfico de contorno de la concentración de calcio, en ppm, durante los meses de diciembre 2008 y enero 2009 en la ciudad de Quito. Datos promedio de cada mes. 
El ion nitrato es un indicador de la neutralización del ácido nítrico. Su aparición en el agua lluvia se explica por la presencia de un alto flujo vehicular y de centrales termoeléctricas que emiten grandes cantidades del precursor $\mathrm{NO}_{x}$. El 33\% de las emisiones anuales de $\mathrm{NO}_{x}$ se producen dentro del límite urbano del DMQ y el $29 \%$ se origina en Guangopolo sitio de las centrales termoeléctricas. A pesar de la distancia, también son fuentes de $\mathrm{NO}_{\mathrm{x}}$, al sur la central de Santa Rosa y al norte la productora de cemento Selva Alegre [7]. De acuerdo a la figura 5 la mayor concentración de nitrato está hacia el centro y sur de la ciudad que se relaciona con áreas industriales y un intenso tráfico vehicular. Además, los contaminantes son acarreados por los vientos que los concentran en el centro de la ciudad.
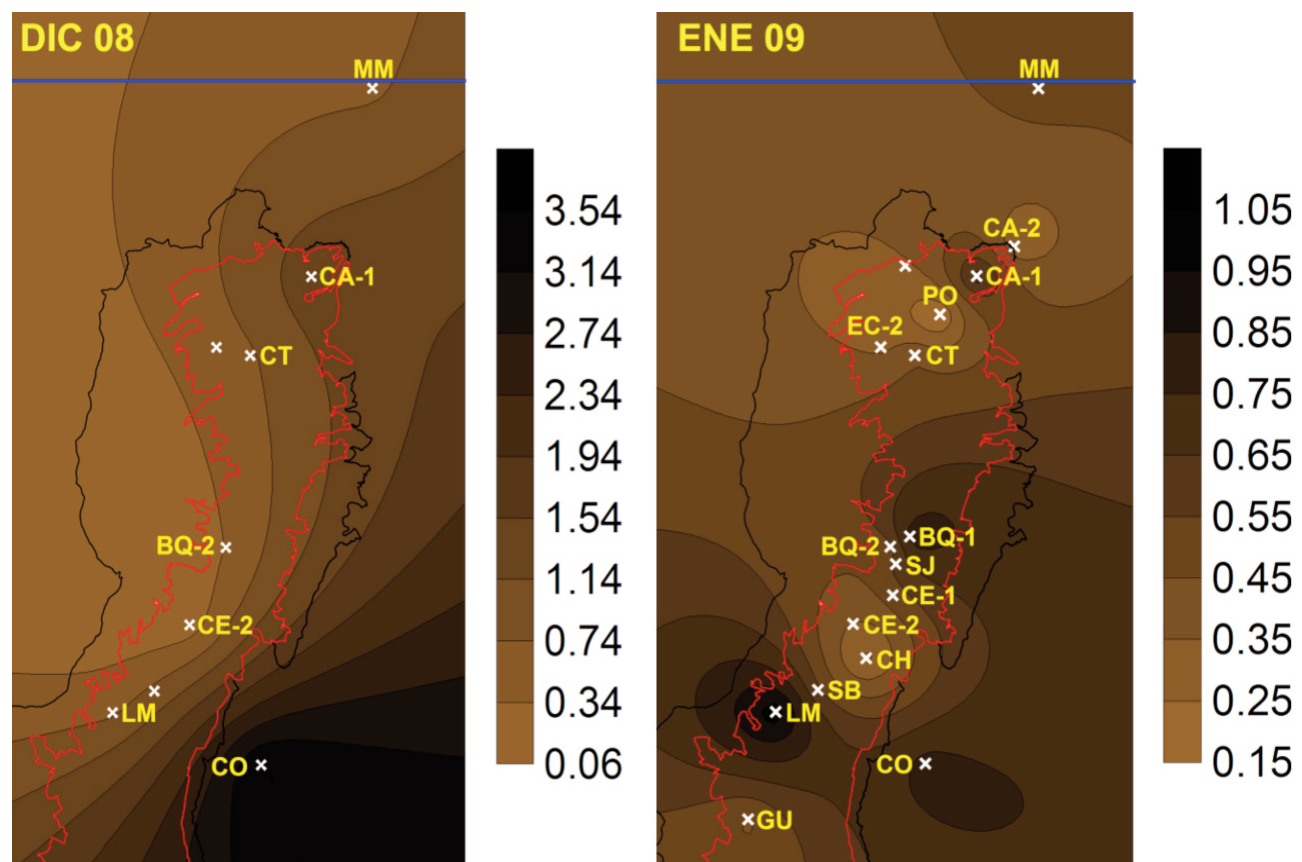

Figura 5. Gráfico de contorno para el ion nitrato, en ppm, en la ciudad de Quito durante los meses de diciembre de 2008 y enero de 2009. Datos promedio mensuales.
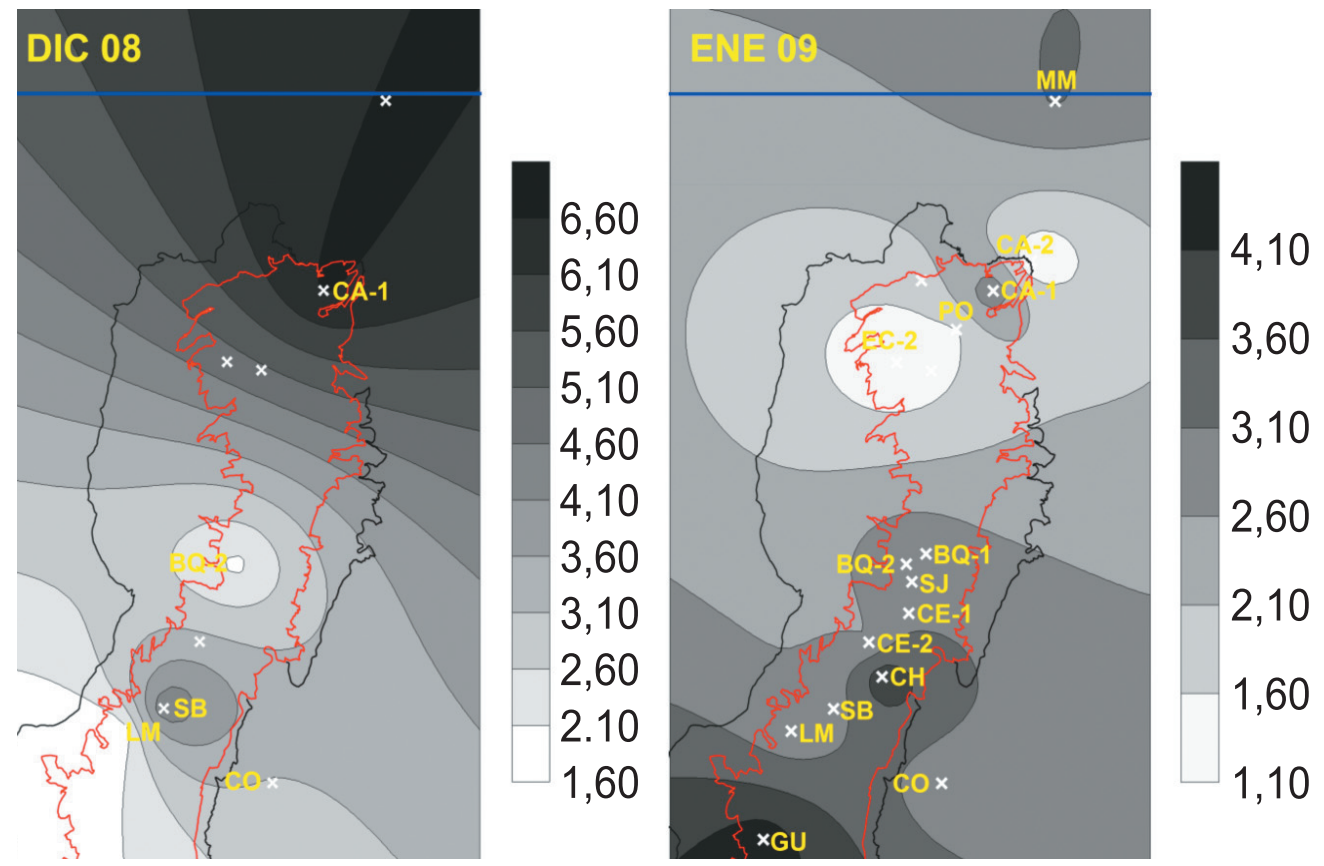

Figura 6. Gráfico de contorno de la concentración de sulfatos, en ppm, para la cuidad de Quito durante los meses de diciembre de 2008 y enero de 2009. Promedio mensual. 
El ion sulfato es el producto de la neutralización del ácido sulfúrico. Los sulfatos se encuentran en la alta densidad industrial y el viento es un factor determinante en la dispersión de los mismos. La fuente primaria para la formación de sulfatos, el óxido de azufre, se emite principalmente de las empresas generadoras de termoelectricidad. El $37,8 \%$ de $\mathrm{SO}_{2}$ se origina dentro del límite urbano del DMQ [7]. La mayor concentración de sulfatos se encontró al norte y al sur de la ciudad (ver Figura 6) que corresponde, al norte, a áreas erosionadas que contribuyen a la neutralización y al sur a zonas de alta densidad industrial.

\section{Conclusiones}

4.1. Los estudios referentes a la composición química de la lluvia son realizados en tiempos prolongados para detectar modificaciones causadas por el incremento de la actividad humana. Este trabajo no busca dar un diagnóstico de la calidad del aire en Quito, ya que para ello es necesario mayor tiempo de estudio.

4.2. La información obtenida, sin embargo, servirá para impulsar un nuevo trabajo mucho más amplio, con mayor número de sitios de muestreo y durante periodos más largos que permitirá observar la evolución de la lluvia ácida en Quito y determinar los correctivos necesarios para evitar su formación.
4.3. Uno de los propósitos de esta investigación fue también involucrar a la ciudadanía en problemas ambientales que les afecta. Esta vez se trabajó con estudiantes universitario para la recolección de las muestras y la medición del $\mathrm{pH}$. En una segunda etapa se trabajará con estudiantes de secundaria para interesarle en la ciencia y en el cuidado del ambiente.

4.4. Se empleó una metodología simple y económica; pero, que brindó datos confiables, lo que permitirá a la ciudadanía participar directamente en problemas ambientales y no ser solamente observadores.

4.5. En el presente estudio pudo apreciarse que aproximadamente el $50 \%$ de la superficie de la ciudad de Quito se encuentra afectada por la lluvia ácida, lo que justifica una investigación mucho más amplia sobre el tema.

\section{Agradecimientos}

Los autores desean agradecer a las autoridades de la Facultad de Ciencias Químicas de la Universidad Central del Ecuador por el apoyo prestado para la realización del proyecto. También, quieren agradecer a todos los estudiantes que voluntaria y desinteresadamente participaron en el proyecto recolectando las muestras y en especial a los estudiantes Diego Lucero, Lenin Vaca y Ximena Vergara por realizar los análisis de las muestras.

6. Referencias

1. Secretaría del Medio Ambiente. (2000). Programa de precipitación ácida de la ZMCM, Informe anual 1999. México: Dirección general de prevención y control de la contaminación.

2. Red Metropolitana de Monitoreo Atmosférico de Quito. (2009). La calidad del aire en Quito Informe anual 2008. Quito: CORPAIRE.

3. Manahan, S. E. (2005). Environmetal chemistry (8 ed.). Florida: CRC Press.

4. GLOBE. (2005). Atmosphere Investigation. Recuperado el 01 de marzo de 2010, de Sitio web del programa GLOBE: www.globe.gov

5. Clarke, A. G., \& Tomlin, A. S. (1999). Chapter 2: The Atmosphere. En R. M. Harrison (Ed.), Understanding Our Environment an Introduction to Environmental Chemistry and Pollution (3 ed.). Cambridge: The Royal Society of Chemistry.

6. Dickson, T. R. (1999). Química enfoque ecológico. (H. Corona, Trad.) México: Editorial Limusa.

7. Red metropolitana de monitoreo atmosférico de Quito. (2009). Inventario de emisiones atmosféricas 2007. Quito: CORPAIRE.

8. Ministerio del Ambiente. (2003). Texto unificado de la legislación ambiental secundaria, Libro VI, Anexo 4 . Recuperado el 03 de marzo de 2010, de Sitio Web del Ministerio del Ambiente del Ecuador: www.ambiente.gov.ec

9. Clesceri, L. S., Greenberg, A. E., \& Eaton, A. D. (Edits.). (1998). Standard Methods for the Examination of water and Wastewater (20 ed.). Maryland: APHA, AWWA, WEF.

10. Yasrebi, J., Saffari, M., Fathi, H., Karimian, N., Moazallahi, M., \& Gani, R. (2009). Evaluation and Comparison of Ordinary Kriging and Inverse Distance Weighting Methods for Prediction of Spatial Variability of Some Soil Chemical Parameters. Research Jomal of Biological Sciences , 4 (1), 93-102.

11. Metzger, P., \& Bermúdez, N. (1996). El Medio Ambiente Urbano en Quito. Quito: Municipio del Distrito Metropolitano de QuitoORSTOM. 